\title{
Factors Related to Successful Energy Transmission of Focused Ultrasound through a Skull : A Study in Human Cadavers and Its Comparison with Clinical Experiences
}

\author{
Na Young Jung, ${ }^{1}$ Itay Rachmilevitch, ${ }^{2}$ Ohad Sibiger, ${ }^{2}$ Talia Amar, $^{2}$ Eyal Zadicario, ${ }^{2}$ Jin Woo Chang ${ }^{1}$ \\ Department of Neurosurgery, Brain Research Institute, Yonsei University College of Medicine, Seoul, Korea \\ InSightec Ltd., ${ }^{2}$ Tirat Carmel, Israel
}

Objective : Although magnetic resonance guided focused ultrasound (MRgFUS) has been used as minimally invasive and effective neurosurgical treatment, it exhibits some limitations, mainly related to acoustic properties of the skull barrier. This study was undertaken to identify skull characteristics that contribute to optimal ultrasonic energy transmission for MRgFUS procedures.

Methods : For ex vivo skull experiments, various acoustic fields were measured under different conditions, using five non-embalmed cadaver skulls. For clinical skull analyses, brain computed tomography data of 46 patients who underwent MRgFUS ablations (18 unilateral thalamotomy, nine unilateral pallidotomy, and 19 bilateral capsulotomy) were retrospectively reviewed. Patients' skull factors and sonication parameters were comparatively analyzed with respect to the cadaveric skulls.

Results : Skull experiments identified three important factors related skull penetration of ultrasound, including skull density ratio (SDR), skull volume, and incidence angle of the acoustic rays against the skull surface. In clinical results, SDR and skull volume correlated with maximal temperature (Tmax) and energy requirement to achieve $\operatorname{Tmax}(p<0.05)$. In addition, considering the incidence angle determined by brain target location, less energy was required to reach Tmax in the central, rather than lateral targets particularly when compared between thalamotomy and capsulotomy $(p<0.05)$.

Conclusion : This study reconfirmed previously identified skull factors, including SDR and skull volume, for successful MRgFUS; it identified an additional factor, incidence angle of acoustic rays against the skull surface. To guarantee successful transcranial MRgFUS treatment without suffering these various skull issues, further technical improvements are required.

Key Words : High-intensity focused ultrasound ablation · Skull · Acoustics.

\section{INTRODUCTION}

Magnetic resonance-guided focused ultrasound (MRgFUS) has been widely applied in various neurological diseases. The main advantage of MRgFUS is minimally invasiveness, due to its ability to penetrate the skull. When the concept of FUS was first introduced in the 1950s, it was impossible to transmit ultrasonic energy through an intact skull; it required an open

\footnotetext{
- Received : December 8, 2018 •Revised : March 4, 2019 •Accepted : March 9, 2019

- Address for reprints : Jin Woo Chang

Department of Neurosurgery, Brain Research Institute, Yonsei University College of Medicine, 50 Yonsei-ro, Seodaemun-gu, Seoul 03722, Korea Tel : +82-2-393-9979, Fax : +82-2-2228-2159, E-mail : JCHANG@yuhs.ac, ORCID : https://orcid.org/0000-0002-2717-0101
} 
window, which was no different from an invasive cranioto$m y^{12-14)}$. The goal of true noninvasiveness has advanced the existing technology to deliver transcranial sonication in deep brain tissue. With improvements of magnetic resonance imaging (MRI) technologies including MR thermometry, FUS successfully became MRgFUS ${ }^{9,17,22,26)}$. In addition, an ultrasonic transducer, which consists of multiple phased array transducer elements, compensates for the ultrasound wave distortion, deflection, and energy absorption of the skull ${ }^{8,9)}$. The phased array transducer creates a tight thermal spot in the targeted location. As a result, MRgFUS has been able to deliver ultrasonic energy without incision or craniotomy. Thus far, high intensity FUS using mid frequency (650 kHz), has been used in a new clinical lesioning procedure to manage a number of neurological disorders, such as essential tremor $(\mathrm{ET})^{2,4,11,15,31)}$, Parkinson's disease (PD) $)^{1,23,25,33)}$, and intractable neuropathic pain ${ }^{10,18,29)}$, as well as neuropsychiatric disorders including obsessive compulsive disorder (OCD $)^{19,21)}$ and major depressive disorder (MDD) ${ }^{20,27)}$. In addition, MRgFUS has become noteworthy in basic research, such as in neuromodulation or blood brain barrier opening. In those applications, low frequency $(230 \mathrm{kHz})$ and low intensity sonications are being used $^{5-7,28,30,32)}$.

Despite its growing popularity, MRgFUS still encounters major obstacles. Optimal targeting should be dependent upon the procedure; however, acquisition of sufficient energy to make the lesion is important to achieve therapeutic success. The skull is a main barrier to the delivery of unimpeded ultrasonic energy to target points in the brain. Elias et al. ${ }^{11}{ }^{11}$ demonstrated that there was difficulty in delivery of sufficient energy for MRgFUS thalamotomy in five of 76 patients with ET; this might have been due to the characteristics of acoustic waves and individual skulls. Chang et al. ${ }^{3)}$ also reported that three of 11 ET patients failed to reach a sufficient temperature increase for tremor management. Essentially, ultrasound waves encounter attenuation, dispersion, and refraction when they pass through the skull, resulting in a significant loss of energy and distortion of the penetrating ultrasound beam ${ }^{24)}$. Although multiple transducers of MRgFUS can boost its focusing ability through the skull ${ }^{16)}$, the beam may encounter unexpected events due to the various skull characteristics of different individuals. The object of this study was to evaluate skull characteristics related to ultrasonic energy transmission and to compare the clinical results of FUS, as well as to improve the efficiency of MRgFUS by complementing its shortcomings.

\section{MATERIALS AND METHODS}

\section{Skull experiments}

Sonication experiments using ex vivo skulls were performed at our institute, in collaboration of the Department of Neurosurgery and the Department of Anatomy. Five human skulls were prepared and modified to measure acoustic fields that could penetrate human calvarias : all brain tissue was removed, including dura mater. The skulls did not undergo embalming processes to reduce the bias caused by the chemical interaction. All fresh skulls were recruited by voluntary donation for the purpose of medical practice, after obtaining consents of the donors and their families. The ultrasound source used was the Exablate 4000 transducer (InSightec, Haifa, Israel) with a radius of $150 \mathrm{~mm}$. The skulls underwent removal of the remaining air inside, and was then placed within a transducer filled with degassed water. A needle hydrophone of the HNC-0400 model (ONDA, Sunnyvale, CA, USA) was inserted into the skull to measure the acoustic pressure in a specific location. The needle hydrophone could be moved and controlled by an attached robotic arm upon the transducers. The acoustic fields were explored in two ways, including two-dimensional (2D) scan from all elements and an acquisition of acoustic correction table (ACT) from each of the elements. The former was to measure acoustic pressure at every point during movement of the hydrophone across the 2D grid when all element of the transducers were acting together. In contrast, when every element was acting separately, the ACT was used to record amplitude and phase of every element, using a hydrophone fixed in one location. The skull was fixed to a mounting frame by weaving in four holes, followed by attachment to the placement frame to experiment at various target points inside the skull. The placement frame was designated with uniformly spaced holes, 1-cm distance between the holes (Fig. 1). As the target position in the skull shifted, various measurements were conducted including energy transmission, phase shifts, and acoustic field shape, in each case. The acoustic measurements were performed using both $230 \mathrm{kHz}$ and $680 \mathrm{kHz}$ transducers. Mid frequency device was used in four skulls and low frequency in five skulls. One skull was not able to undergo experiments in low frequency transducer due to 
time limitations of the non-embalmed skull condition.

\section{Clinical applications}

Retrospective review was conducted using sonication data of 46 patients who underwent MRgFUS ablation from February 2013 to August 2017. They were classified with four disease entities : ET, OCD, PD, and MDD. Eighteen of 46 patients underwent unilateral MRgFUS thalamotomy for ET, targeting the left ventrointermediate nucleus of the thalamus (Vim). Nine PD patients experienced unilateral MRgFUS pallidotomy to control their severe dyskinesia, targeting the left posteroventral globus pallidus interna (Gpi). In addition, psychosurgical procedures were performed in 19 patients (16 OCD and three MDD) at the bilateral anterior limb of the internal capsule (ALIC) using noninvasive sonication. Detailed target coordinates are described in Table 1.
The entire MRgFUS procedure was performed in a 3-T MRI scanner (GE Medical Systems, Milwaukee, WI, USA) using the Exablate 4000 device (InSightec). After stereotactic frame fixation to the skull and assembly of the MRgFUS system, diagnostic MRI scans were acquired and the coordinates of target points were set to focus ultrasonic energy. Prior to producing permanent thermal lesions, the size and location of a real thermal spot was evaluated using subthreshold lowpower sonication. Then, acoustic power and energy were steadily increased to reach a sufficient temperature to generate a permanent lesion under the guidance of MRI and MR thermometry. The neurological status of the patients was repeatedly assessed during the cooling time between each sonication.

Other calculations regarding the skulls were performed in an automatic program, mainly using computed tomography

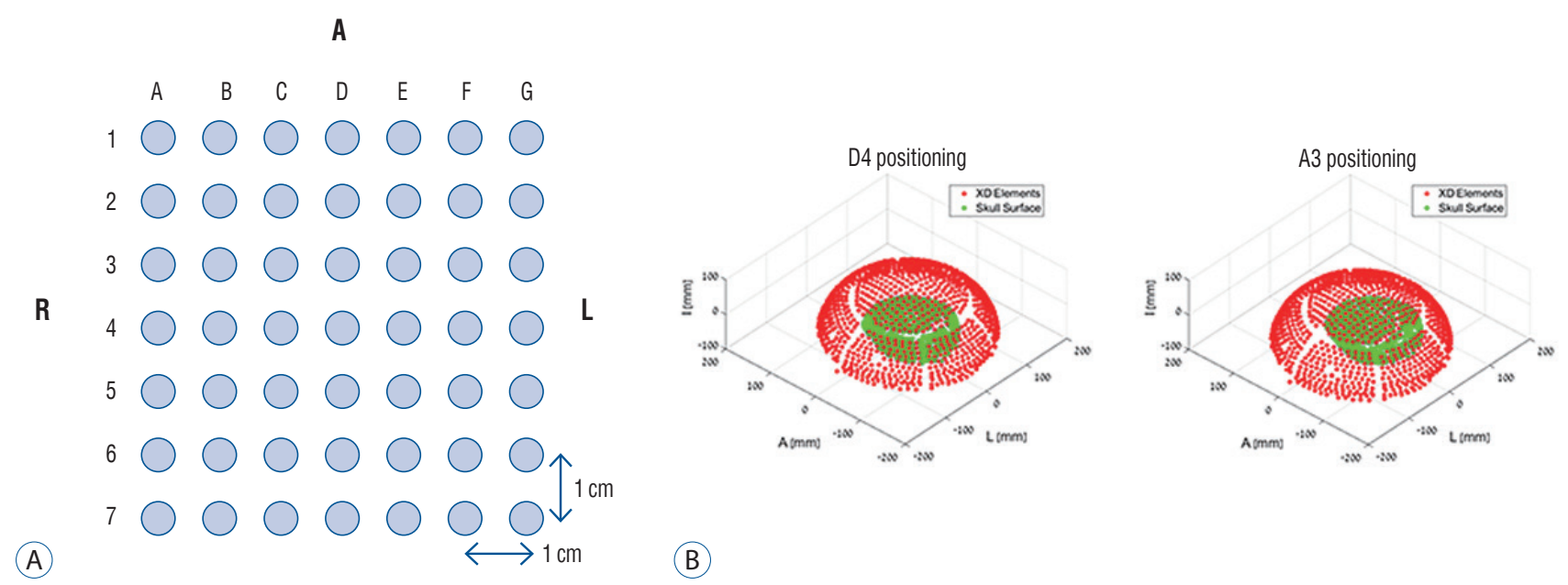

Fig. 1. Skull mounting to the placement frame. Each hole indicates a specific location inside the skull. D4, for example, is the center and $A 3$ is a target, $3 \mathrm{~cm}$ right and $1 \mathrm{~cm}$ anterior from the center (A). Therefore, D4 indicates that the skull is located in the center of the transducer, while A3 indicates that it is in a more lateral location (B).

Table 1. Target coordinates for magnetic resonance-guided focused ultrasound ablation

\begin{tabular}{|c|c|c|}
\hline Disease & Target & Coordinates \\
\hline Essential tremor & Ventrointermediate nucleus of thalamus & $\begin{array}{l}14-15 \mathrm{~mm} \text { lateral to the MCP and } 6-7 \mathrm{~mm} \text { anterior to } \\
\text { the posterior commissure, and at the ICL level }\end{array}$ \\
\hline $\begin{array}{l}\text { Obsessive-compulsive disorder and major } \\
\text { depressive disorder }\end{array}$ & Anterior limb of internal capsule & $\begin{array}{l}7 \mathrm{~mm} \text { anterior to the anterior commissure and at the } \\
\mathrm{ICL} \text { level, extending } 2-3 \mathrm{~mm} \text { along the capsule from } \\
\text { a coronal view }\end{array}$ \\
\hline Parkinson's disease & Posteroventral portion of globus pallidus interna & $\begin{array}{l}20 \mathrm{~mm} \text { lateral and 3-4 mm anterior to the MCP, and } \\
3-4 \mathrm{~mm} \text { inferior to the ICL }\end{array}$ \\
\hline
\end{tabular}

MCP : midcommissural point, ICL : intercommissural line 
(CT) scanning. Skull density ratio (SDR) was calculated by CT Density Analysis Tool (InSightec) and skull volume or thickness was performed by using a three-dimensional image software (Aquarius version 4.4; TeraRecon, Foster City, CA, USA).

Based on the experimental conditions identified in the cadaveric experiments, the clinical patients were classified into several groups, according to sonication parameters and clinical characteristics of each patient's skull. We performed comparative analysis of the sonication procedures between ex vivo skulls and in vivo skulls, focusing on effective conditions for ultrasonic penetration through the human skull. Written informed consent was obtained from all included patients prior to the procedures. This study received full approval from the Institutional Review Board of Yonsei University College of Medicine (1-2018-0087).

\section{Statistical analysis}

All statistical analyses were performed using SPSS version 23 (IBM Corporation, New York, NY, USA). Linear regression methods were used to determine the correlation between skull- or sonication-related factors and maximal temperature (Tmax) or energy applied for Tmax rise. The threshold for statistical significance was set at $p<0.05$. Any comparisons among the three different target groups were selectively analyzed by analysis of variance or KruskalWallis test, according to the normality of the data. For the post analysis, the statistical significance level was corrected using Bonferroni's method $(p<0.017)$.

\section{RESULTS}

\section{Participants}

Skull experiments were performed in five adult cadaver skulls that were not processed by chemical agents for preservation. The mean skull thickness was $6.6 \pm 0.9 \mathrm{~mm}$ and the volume was $302.1 \pm 41.3 \mathrm{~cm}^{3}$. The average SDR was $0.47 \pm 0.13$.

The clinical studies constituted 46 patients, including 18 patients with ET, nine with PD, 16 with OCD and three MDD. Their mean age was 51.4 years (range, 21-75). There were more men than women $(29: 17)$. The characteristics of in vivo skulls were as follow : mean thickness of $5.6 \pm 1.2 \mathrm{~mm}$, skull volume of $317.1 \pm 52.3 \mathrm{~cm}^{3}$, and SDR of $0.57 \pm 0.10$. The detailed demographics related to skull and MRgFUS procedure of the clinical patients are described in Table 2.

\section{Skull density ratio}

SDR indicates the ratio of Hounsfield unit value on CT between the marrow and cortical bone, which is originally calculated as $<1$ (Fig. 2). A lower SDR indicates a relatively higher cortical bone density than bone marrow, which signifies a larger difference of acoustic impedance between two types of tissues. This difference can affect the degree to which ultrasonic waves are transmitted.

In the experimental setting, five skulls showed a positive correlation between SDR and relative intensity (RI). The RI of ultrasound is represented as the square value of integral calculus, consisting of the relative amplitude of ultrasound, both in

Table 2. Demographics of patients who underwent magnetic resonance-guided focused ultrasound ablation

\begin{tabular}{lccr} 
& Essential tremor & $\begin{array}{c}\text { Obsessive-compulsive disorder } \\
\text { and major depressive disorder }\end{array}$ & Parkinson's disease \\
\hline Mean age (years) & $65.4(52-75)$ & $35.0(21-56)$ & $58.1(43-74)$ \\
Sex (men : women) & $15: 3$ & $10: 9$ & $4: 5$ \\
Skull density ratio & $0.57 \pm 0.1$ & $0.57 \pm 0.1$ & $0.61 \pm 0.1$ \\
Skull thickness $(\mathrm{mm})$ & $5.1 \pm 0.9$ & $5.9 \pm 1.4$ & $5.9 \pm 0.7$ \\
Skull volume $\left(\mathrm{cm}^{3}\right)$ & $319.2 \pm 64.3$ & $317.8 \pm 47.6$ & $311.3 \pm 38.1$ \\
Skull area $\left(\mathrm{cm}^{2}\right)$ & $342.6 \pm 19.8$ & $296.8 \pm 57.3$ & $315.9 \pm 78.5$ \\
Number of sonications & $16.6(8-22)$ & $27.2(12-43)$ & $21.3(10-25)$ \\
Mean maximal energy delivered $(\mathrm{J})$ & $15977.5 \pm 6712.2$ & $23907.7 \pm 9690.5$ & $20001.1 \pm 7024.2$ \\
Peak temperature $\left({ }^{\circ} \mathrm{C}\right)$ & $57.4 \pm 2.2$ & $56.6 \pm 4.9$ & $58.1 \pm 7.1$ \\
Number of sonications above $54^{\circ} \mathrm{C}$ & $7.4 \pm 2.5$ & $6.5 \pm 5.0$ & $4.4 \pm 3.7$ \\
\hline
\end{tabular}

Values are presented as mean (range) or mean \pm standard deviation unless otherwise indicated 


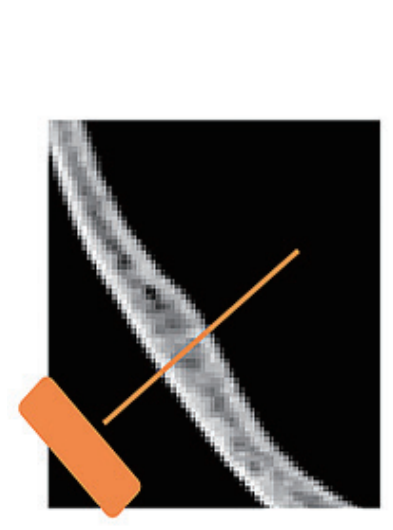

XD element

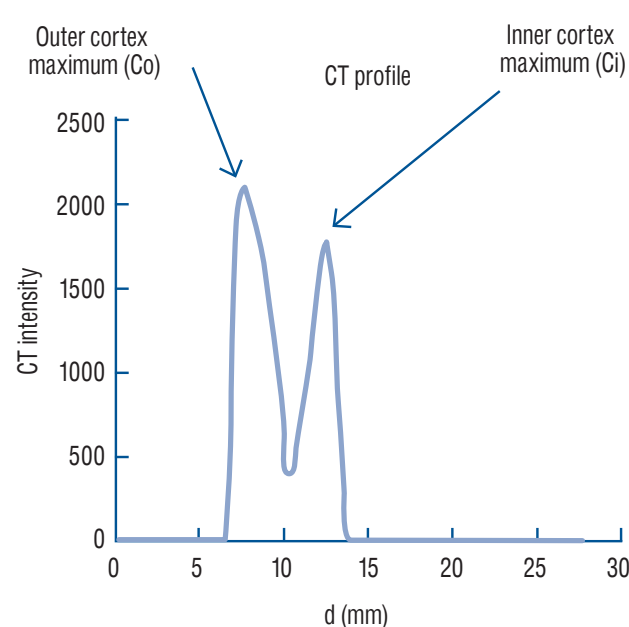

Marrow minimum (m)

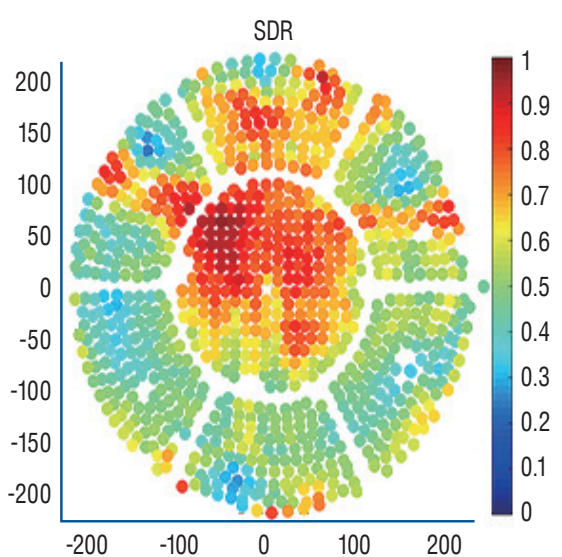

(B)

(A)

Fig. 2. Reference drawing showing how to measure the SDR on brain computed tomography (A) and how to average SDR on all elements of Exablate 4000 (InSightec, Tirat Carmel, Israel) (B). CT : computed tomography, SDR: skull density ratio.

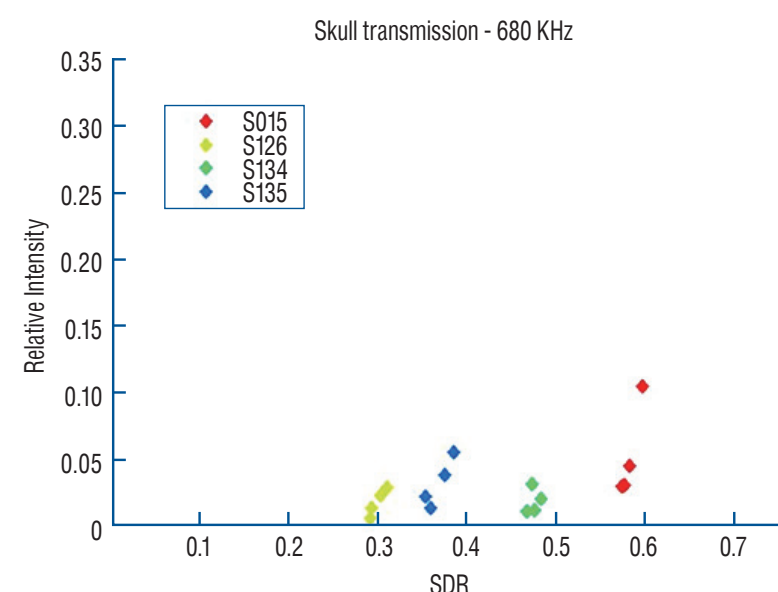

(A)

(C)

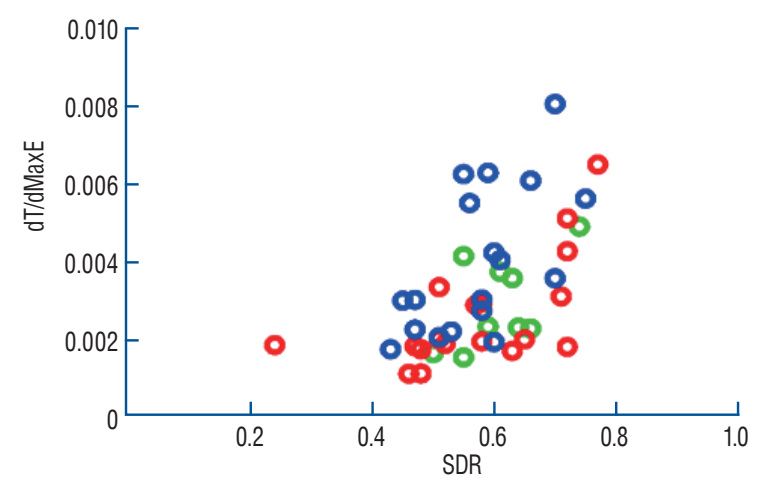

Skull transmission - $230 \mathrm{KHz}$

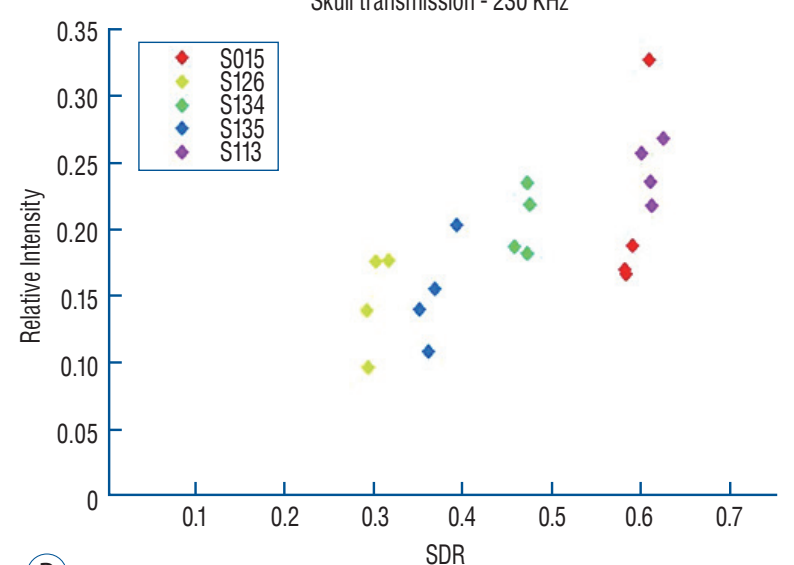

(B)
- Thalamotomy
- Capsulotomy
Pallidotomy

Fig. 3. Positive correlation between SDR and relative intensity of ultrasonic energy through the skull. These correlations were similarly identified in conditions with either mid frequency (A) or low frequency (B). In the clinical results, there was a positive correlation between SDR and temperature rise per unit energy, revealing greater transmitted energy (C).SDR: skull density ratio, $\mathrm{dT} / \mathrm{dMaxE}$ : temperature rise per unit energy. 
plain water and after skull penetration.

Relative intensity $(\mathrm{RI})=\left\{\sum_{\text {elements }}\left(\frac{\text { a skull }}{\text { a water }}\right)\right\}^{2}$

This can be interpreted as the definition of transmission efficiency. Although the RI of mid frequency sonication only became one third of the results of low frequency experiments, similar tendencies were maintained in association with SDR (Fig. 3).

In the clinical results, SDR was significantly positively correlated with peak temperature $\left(p<0.001, \mathrm{r}^{2}=0.313, \mathrm{y}=43.156+24.326 \mathrm{x}\right)$ and the magnitude of temperature increase above $54^{\circ} \mathrm{C}(p<0.001$, $\left.\mathrm{r}^{2}=0.317, \mathrm{y}=-6.046+21.584 \mathrm{x}\right)$. Although none of the skull factors were correlated with SDR, independent skull characteristics showed an inverse correlation with the maximal energy delivered to reach peak temperature $\left(p=0.002, \mathrm{r}^{2}=0.198, y=41512.5-\right.$ $37132.5 \mathrm{x})$. In the clinical setting, the magnitude of temperature increase per unit energy was analyzed as a function of transmission efficiency, since it is difficult to place the hydrophone into the human brain and measure the actual RI of the ultrasonic energy. The high SDR was correlated with increases in the magnitude of temperature increase per unit energy $\left(p<0.001, \mathrm{r}^{2}=0.285\right.$, $y=-0.002+0.008 x)$. Thus, high SDR could make it easy to achieve sufficient temperature and subsequent lesions, using relatively low energy. Results from both ex vivo and in vivo skulls both indicated that high SDR is more efficient at energy transmission and at raising temperatures to make permanent lesions in the brain.

\section{Skull volume and thickness}

Other potential factors of the skull that impeded ultrasonic energy transfer included skull volume, thickness, and skull area. Among these factors, Tmax was negatively correlated with skull thickness $\left(p=0.006, \mathrm{r}^{2}=0.162, \mathrm{y}=65.792-1.520 \mathrm{x}\right)$ and skull volume ( $\left.p=0.043, \mathrm{r}^{2}=0.090, \mathrm{y}=65.501-0.026 \mathrm{x}\right)$. Skull area had no significant relationship with Tmax ( $p=0.161)$. In terms of maximal energy requirement to achieve Tmax, only skull volume was closely related with energy delivery required to reach Tmax ( $p=0.045, \mathrm{r}^{2}=0.088, \mathrm{y}=3001.7+43.8 \mathrm{x}$ ) (Fig. 4).

\section{Incidence angle}

Theoretically, energy reflection and transmission of ultrasound are correlated with the respective acoustic impedances of the different materials and the incidence angle of ultrasound wave. In terms of the incidence angle, the mean values of the angles are higher for targets located laterally than for those in the center position. In the skull experiments, lateral targets showed a much more unfocused ultrasound than those in the center position. However, there was no difference in the ability of energy to focus at a single point, when targets were optimally corrected (Fig. 5). In addition, we set 25 degrees as a standard value because the ultrasound beam in the Exablate system showed steeply increased reflection and decreased transmission with incidence angles $>25$ degrees. Considering that incidence beam angles within 25 degrees are efficient for transmission through the skull, as the target location moves further from the center, the fewer number of $\mathrm{cu}^{-}$
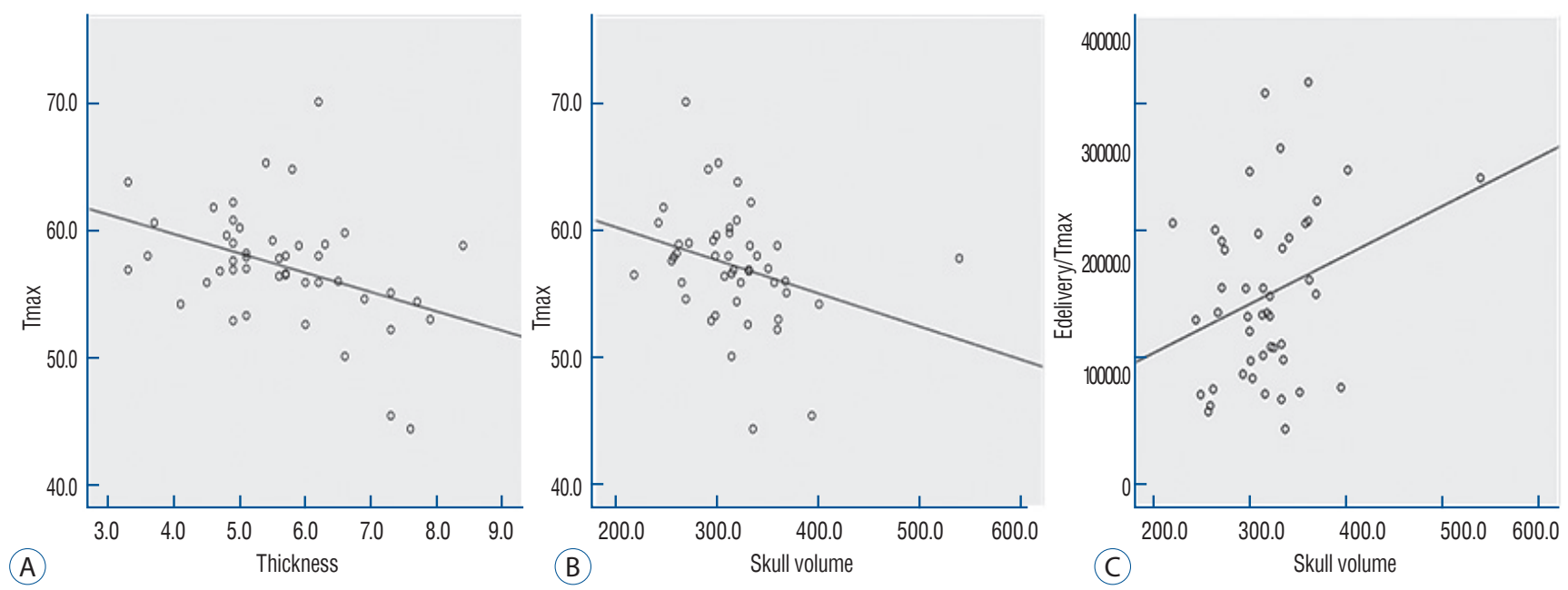

Fig. 4. Linear regression of skull thickness, skull volume, and Tmax (A and B). Skull volume is also correlated with maximal energy delivery to reach maximal temperature (C). Tmax: maximal temperature, Edelivery/Tmax: energy delivered to reach maximal temperature. 
mulative transducer elements can be used for successful sonication (Fig. 6A). When comparing clinical data across three different targets, it was confirmed that Vim exhibited the most ultrasound elements that could be effective for therapeutic sonication, and was sequentially followed by ALIC and Gpi (Fig. 6B).

As the incidence angle became larger, the energy transmission through the skull was less effective in both settings at low and mid frequencies, which appeared more clearly in skull conditions with low SDRs (Fig. 7A and B). Particularly when the outer incidence angle was $>25$ degrees, amplitude transmission sharply decreased in circumstances where SDR was less than 0.6. These experimental results of energy transmission were consistent with clinical results. The average of inci-

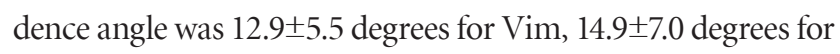
ALIC, and 16.5 \pm 5.9 degrees for Gpi. Notably, the ultrasonic energy required for Tmax increase was significantly different

Low incidence angle (D4)
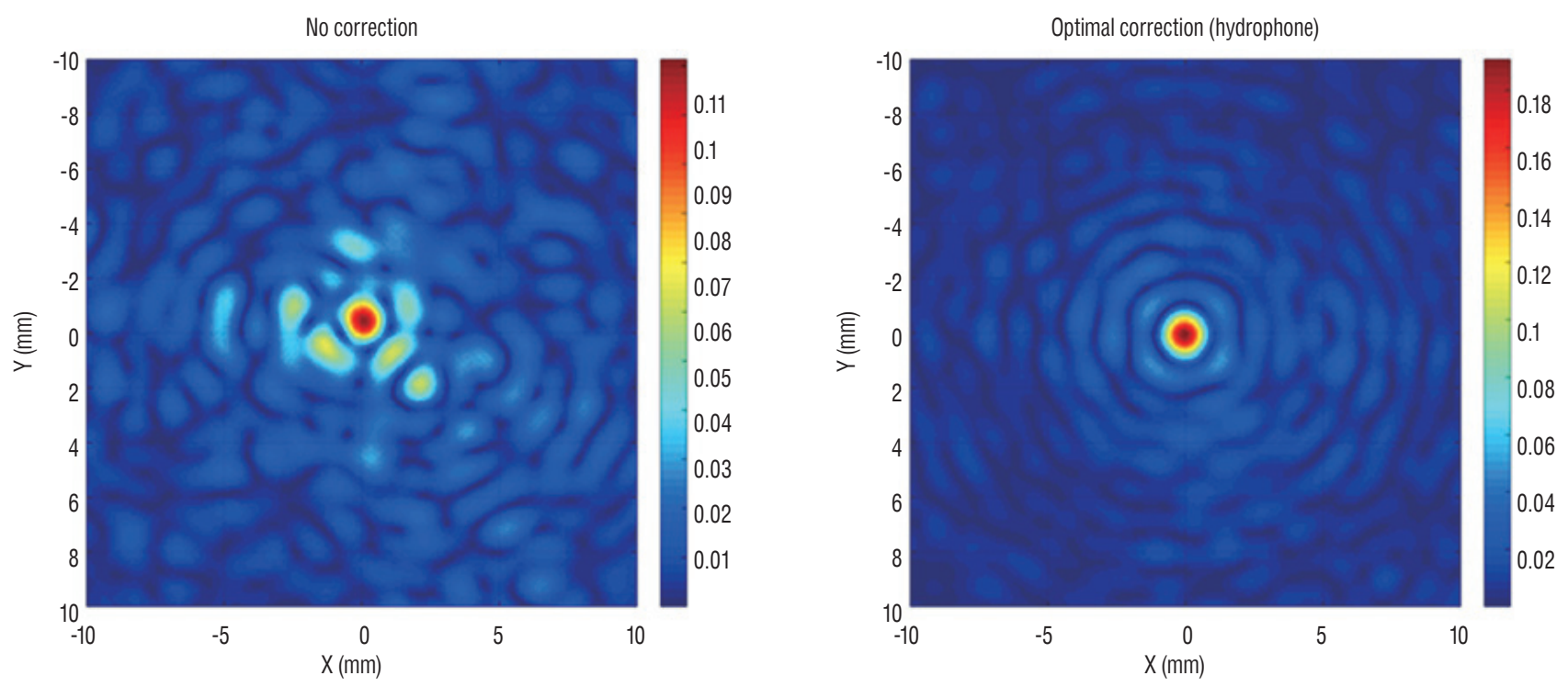

High incidence angle (A3)
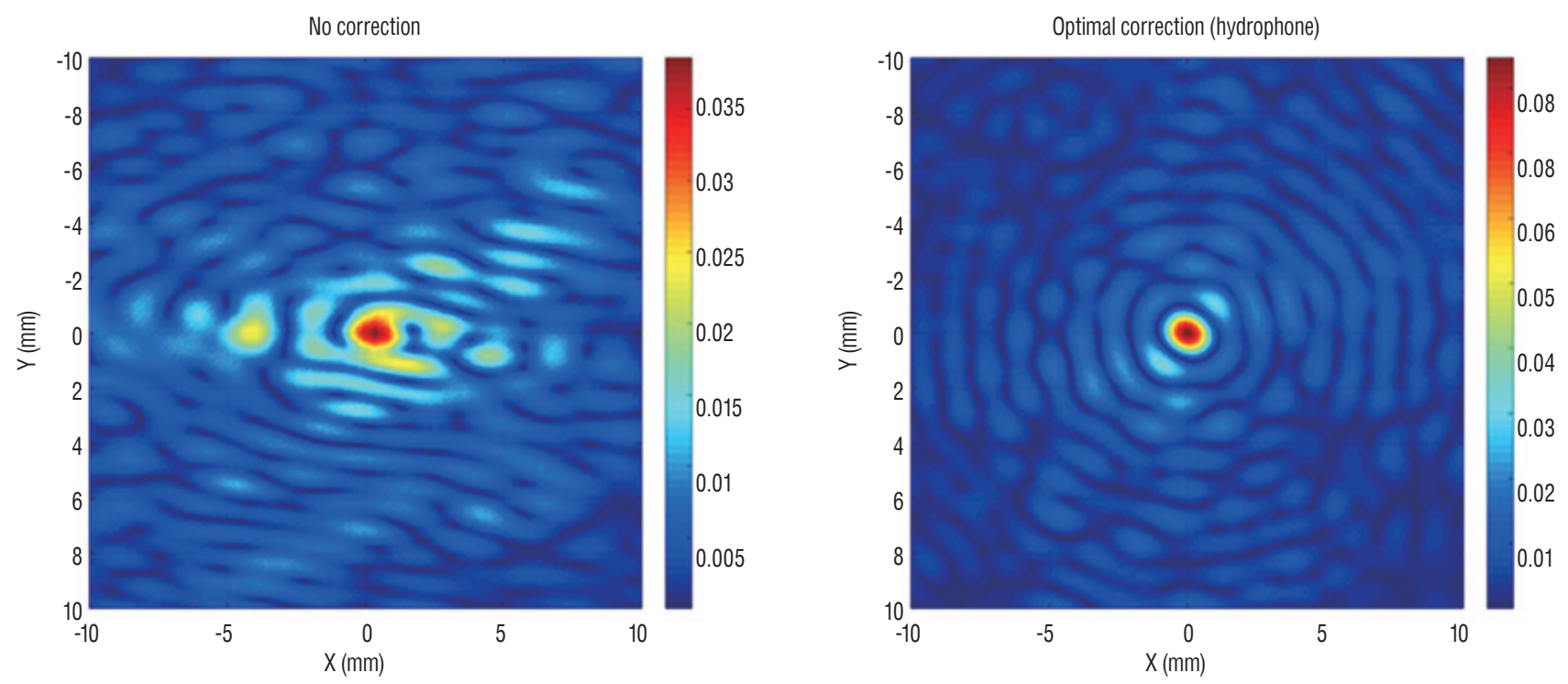

Fig. 5. Two-dimensional scan showing appearance of acoustic rays when focusing on a specific target location. A center location (D4) revealed more centralized acoustic rays than a laterally located target with a high incidence angle. However, acoustic beam fields demonstrated a similarly centralized shape during optimal correction of acoustic rays by hydrophone. 
among the groups : $15977.5 \pm 6712.2 \mathrm{~J}$ for thalamotomy, 23907.7 \pm 9690.5 for capsulotomy, and 20001.1 \pm 7024.2 for pallidotomy ( $p=0.022$ ). In particular, post hoc analysis (Bonferroni's method) comparing two groups showed thalamotomy consumed much less energy to reach peak temperature and lesioning, compared with capsulotomy $(p=0.015)$. It demonstrated a similar result when compared with pallidotomy, but this was not statistically significant ( $p=0.698$ ) possibly due to the small number of cases of pallidotomy. This has reconfirmed that the efficiency of the clinical focused ultrasound can vary depending on the target location.

Interestingly, cases with SDR $\geq 0.6$ showed slightly improved amplitude transmission at incidence angles $>25$ degrees (Fig. 7C), indicating that high SDRs are less affected by the influence of the incidence angle. In addition, low frequency ultrasound showed better amplitude transmission than
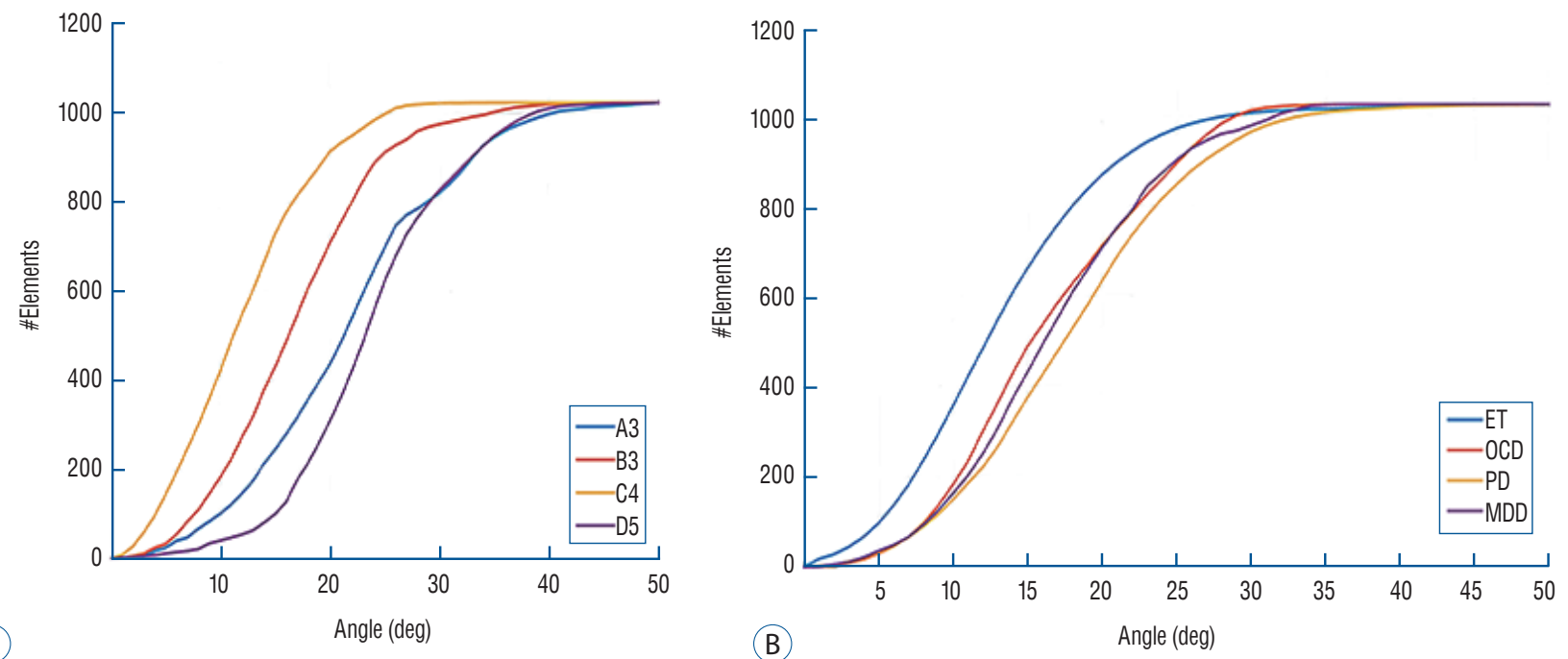

Fig. 6. Cumulative number of sonication elements according to incidence angle. When setting the incidence angle $<25$ as the standard cut-off value, a laterally positioned target acquires relatively fewer elements that could be usable for energy transmission. Similar patterns are confirmed in the skull experiments (A) and clinical data (B). ET : essential tremor, OCD : obsessive compulsive disorder, PD : Parkinson's disease, MDD : major depressive disorder.
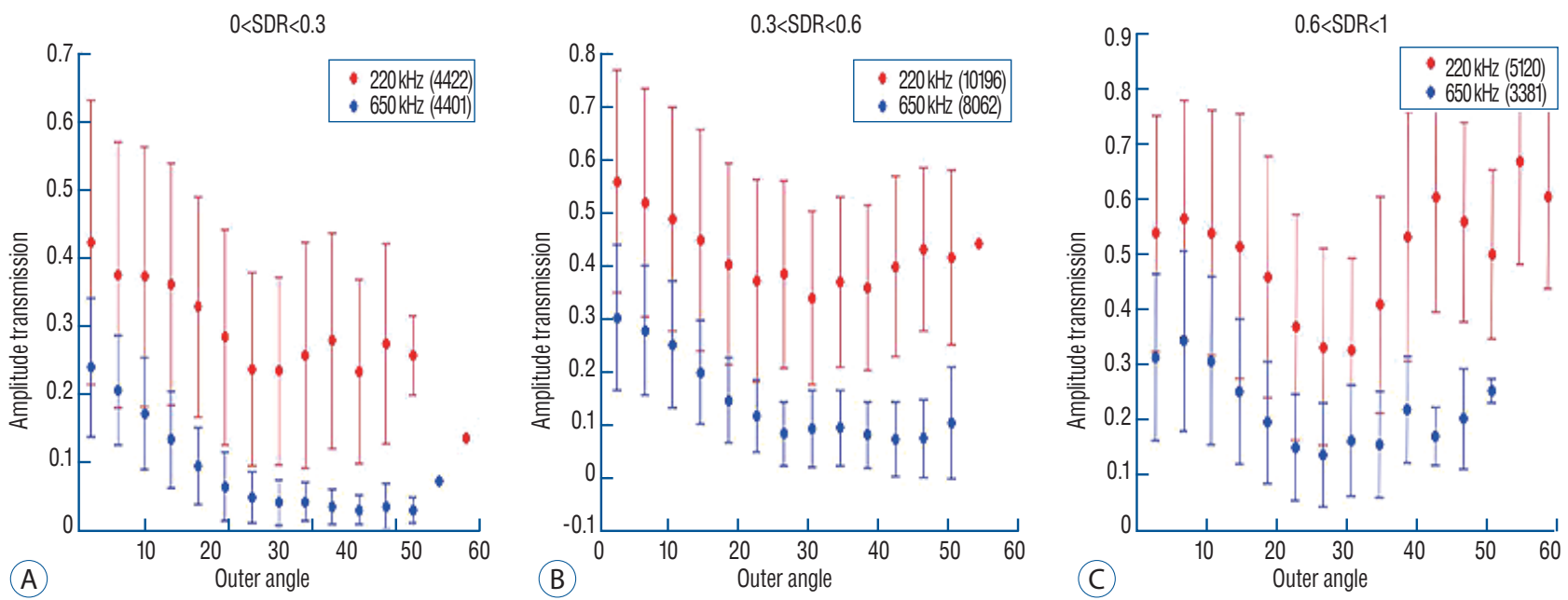

Fig. 7. Mean value of transmission amplitude according to the incidence angle of acoustic rays. When dividing the three groups by SDR value, energy transmission through the skull generally exhibited less effective as the incidence angle become larger (A-C). Interestingly, cases with SDR $\geq 0.6$ showed slightly improved amplitude transmission at incidence angles $>25$ degrees (C), indicating that high SDRs are less affected by the influence of the incidence angle. Moreover, low frequency ultrasound demonstrated much better transmission than mid frequency in all SDR conditions. SDR: skull density ratio. 
mid frequency ultrasound in all SDR conditions and/or larger incidence angles. These results will play an important basic role for selecting clinical target and calibrating the technology.

\section{DISCUSSIONS}

Although the clinical results of transcranial MRgFUS treatment are generally feasible and safe f,2, $, 411,19-21,23)$, it is not yet clear whether this procedure can be successfully performed in all patients with variable conditions, or whether it can be applied to any location in the brain. The ultimate goal of this study was to investigate the skull factors related to transcranial ultrasound to improve the effectiveness of MRgFUS.

SDR has been used as an important factor in making treatment decisions. It is well known that a lower SDR is less efficient for generating lesions with MRgFUS, possibly due to lower energy transmission by reflection or scattering of the ultrasound waves. This has been reaffirmed in our study. Our previous study rated the SDR standard as 0.45 , because SDR $>0.45$ predicted a lower energy requirement for generating a sufficient temperature increase in the target $\operatorname{area}^{3)}$. However, SDR may vary between institutes according to different CT settings, and there is not an absolute cutoff value for selecting suitable candidates. Fortunately, the new version of the Exablate 4000 device 7.0 (InSightec, Tirat Carmel, Israel) measures SDR automatically. Therefore, it is necessary to gather a large amount of multicenter data and establish common SDR criteria as suitable indications for MRgFUS.

Even though aberration correction methods have alleviated many issues of ultrasound technology, non-spherical skull shape and non-centric target locations can be still challenging for MRgFUS ${ }^{8}$. In the previous study, we observed that the incidence angle was insufficient to cause reflection and defocusing of ultrasound, and that it was not correlated with increased temperature ${ }^{3}$. In this study, we found that a more lateral target acquired relatively fewer ultrasound elements that are useful for transferring energy. As a result, the energy requirement for Tmax was less in cases undergoing thalamotomy (more central locations) than in those undergoing capsulotomy. Pallidotomy, the most lateral target location, also used more energy than thalamotomy, but the difference was not statistical significant. This might result from the small number of included cases or the confounding effects of better SDR conditions in patients undergoing pallidotomy. In this surgical approach, it is best to be as careful as possible that the target location is centered in the stereotactic frame. In order to expand its application to other clinical fields where treatment position can be located more laterally or superficially, additional studies must be performed focusing on model correction according to target location, in the future.

Interestingly, the results of this study demonstrated potential that high SDR may compensate for energy insufficiency related to the incidence angle. Additionally, low frequency may be less affected by skull characteristics than mid frequen$\mathrm{cy}$ is, in terms of energy transmission. If the focusing range for a target could be appropriately adjusted, low frequency sonication can be used as a very good supplement at this clinical stage. Excluding these skull-related limitations, increasing energy deposition in the target area using microbubbles can be a task for researchers.

This study has several limitations. Above all, the variable characteristics of other tissues, including brain parenchyma, scalp, or muscles were not considered. Volume, solid/water contents, and the presence of calcification in brain tissues are factors that are likely to disturb ultrasound energy by absorption, scattering, or attenuation. Scalp thickness and distribution within the soft tissue organization are a necessary component of future research. Nevertheless, similar results between ex vivo and in vivo skull studies indicated that skull-related factors are one of the most influential barriers to overcome in the broader application of MRgFUS. Notably, a small number of cases have fundamental limitations not to escape statistical error. Further investigations using large scale, multicenter data analyses are necessary.

\section{CONCLUSION}

MRgFUS is one of the potential steps towards an ideal noninvasive procedure for neurological disorders. This study reconfirmed that skull-related factors, such as SDR and skull volume, could be important factors to consider in overcoming the limitations presented by MRgFUS at the current clinical stage. In addition, the incidence angle of acoustic rays associated with different target locations is another key factor for successful treatment by MRgFUS. Technological developments are necessary to correct the sophistication of the cur- 
rent apparatus and to cope with a variety of skull structures. These advancements could guarantee successful MRgFUS treatment involving various targets in the brain.

\section{CONFLICTS OF INTEREST}

Dr. Chang reports grants from the Michael J. Fox Foundation (New York, USA) and the Focused Ultrasound Surgery Foundation (Virginia, USA), during the conduct of the clinical study. Other authors have no conflict of interest to declare.

\section{INFORMED CONSENT}

Informed consent was obtained from all individual participants included in this study.

\section{AUTHOR CONTRIBUTIONS}

\author{
Conceptualization : JWC, EZ \\ Data curation : OS, TA \\ Formal analysis : NYJ, IR, TA \\ Funding acquisition : JWC \\ Methodology: NYJ, IR \\ Project administration : JWC \\ Visualization : OS, TA \\ Writing - original draft : NYJ \\ Writing - review \& editing : JWC
}

\section{- Acknowledgements}

This study was previously presented as poster in the 6th International Symposium on Focused Ultrasound 2018.

\section{References}

1. Bond AE, Shah BB, Huss DS, Dallapiazza RF, Warren A, Harrison MB, et al. : Safety and efficacy of focused ultrasound thalamotomy for patients with medication-refractory, tremor-dominant parkinson disease: a randomized clinical trial. JAMA Neurol 74 : 1412-1418, 2017

2. Chang WS, Jung HH, Kweon EJ, Zadicario E, Rachmilevitch I, Chang JW : Unilateral magnetic resonance guided focused ultrasound thalamotomy for essential tremor: practices and clinicoradiological outcomes. J Neurol Neurosurg Psychiatry $86:$ 257-264, 2015

3. Chang WS, Jung HH, Zadicario E, Rachmilevitch I, Tlusty T, Vitek S, et al. : Factors associated with successful magnetic resonance-guided focused ultrasound treatment: efficiency of acoustic energy delivery through the skull. J Neurosurg 124 : 411-416, 2016

4. Chazen JL, Sarva H, Stieg PE, Min RJ, Ballon DJ, Pryor KO, et al. : Clinical improvement associated with targeted interruption of the cerebellothalamic tract following MR-guided focused ultrasound for essential tremor. J Neurosurg $129:$ 315-323, 2018

5. Chen PY, Hsieh HY, Huang CY, Lin CY, Wei KC, Liu HL : Focused ultrasound-induced blood-brain barrier opening to enhance interleukin-12 delivery for brain tumor immunotherapy: a preclinical feasibility study. J Transl Med $13:$ 93, 2015

6. Choi JJ, Wang S, Brown TR, Small SA, Duff KE, Konofagou EE : Noninvasive and transient blood-brain barrier opening in the hippocampus of alzheimer's double transgenic mice using focused ultrasound. Ultrason Imaging 30 : 189-200, 2008

7. Chu PC, Liu HL, Lai HY, Lin CY, Tsai HC, Pei YC : Neuromodulation accompanying focused ultrasound-induced blood-brain barrier opening. Sci Rep 5 : 15477, 2015

8. Clement GT, White J, Hynynen K : Investigation of a large-area phased array for focused ultrasound surgery through the skull. Phys Med Biol 45 : 1071-1083, 2000

9. Clement $\mathrm{GT}$, Hynynen $\mathrm{K}$ : A non-invasive method for focusing ultrasound through the human skull. Phys Med Biol 47 : 1219-1236, 2002

10. Dobrakowski PP, Machowska-Majchrzak AK, Labuz-Roszak B, Majchrzak KG, Kluczewska E, Pierzchala KB : Mr-guided focused ultrasound: a new generation treatment of parkinson's disease, essential tremor and neuropathic pain. Interv Neuroradiol 20 : 275-282, 2014

11. Elias WJ, Lipsman N, Ondo WG, Ghanouni P, Kim YG, Lee W, et al. : A randomized trial of focused ultrasound thalamotomy for essential tremor. N Engl J Med 375 : 730-739, 2016

12. Fry FJ, Ades HW, Fry WJ : Production of reversible changes in the central nervous system by ultrasound. Science $127: 83-84,1958$

13. Fry WJ, Mosberg WH Jr, Barnard JW, Fry FJ : Production of focal destructive lesions in the central nervous system with ultrasound. J Neurosurg $11: 471-478,1954$

14. Fry WJ, Barnard JW, Fry EJ, Krumins RF, Brennan JF : Ultrasonic lesions in the mammalian central nervous system. Science 122 : 517-518, 1955

15. Gallay MN, Moser D, Rossi F, Pourtehrani P, Magara AE, Kowalski M, et al. : Incisionless transcranial mr-guided focused ultrasound in essential tremor: cerebellothalamic tractotomy. J Ther Ultrasound 4 : 5, 2016

16. Hynynen K, Clement GT, McDannold N, Vykhodtseva N, King R, White $P J$, et al. : 500-element ultrasound phased array system for noninvasive focal surgery of the brain: a preliminary rabbit study with ex vivo human skulls. Magn Reson Med 52 : 100-107, 2004

17. Ishihara Y, Calderon A, Watanabe H, Okamoto K, Suzuki Y, Kuroda K, et al. : A precise and fast temperature mapping using water proton chemical shift. Magn Reson Med 34 : 814-823, 1995

18. Jeanmonod D, Werner B, Morel A, Michels L, Zadicario E, Schiff G, et al. : 
Transcranial magnetic resonance imaging-guided focused ultrasound: noninvasive central lateral thalamotomy for chronic neuropathic pain.

Neurosurg Focus 32 : E1, 2012

19. Jung HH, Kim SJ, Roh D, Chang JG, Chang WS, Kweon EJ, et al. : Bilateral thermal capsulotomy with MR-guided focused ultrasound for patients with treatment-refractory obsessive-compulsive disorder: a proof-ofconcept study. Mol Psychiatry 20 : 1205-1211, 2015

20. Kim M, Kim CH, Jung HH, Kim SJ, Chang JW : Treatment of major depressive disorder via magnetic resonance-guided focused ultrasound surgery. Biol Psychiatry 83 : e17-e18, 2018

21. Kim SJ, Noh DY, Jung HH, Chang WS, Chang JW, Kim H : PT620. A pilot study of bilateral thermal capsulotomy with focused ultrasound for treatment-refractory (MRgFUS) obsessive-compulsive disorder. Int J Neuropsychopharmacol 19(Suppl 1) : 27-28, 2016

22. Kuroda K, Oshio K, Chung AH, Hynynen K, Jolesz FA : Temperature mapping using the water proton chemical shift: a chemical shift selective phase mapping method. Magn Reson Med 38 : 845-851, 1997

23. Martínez-Fernández R, Rodríguez-Rojas R, Del Álamo M, HernándezFernández F, Pineda-Pardo JA, Dileone $M$, et al. : Focused ultrasound subthalamotomy in patients with asymmetric parkinson's disease: a pilot study. Lancet Neurol 17 : 54-63, 2018

24. Mueller JK, Ai L, Bansal P, Legon W : Numerical evaluation of the skull for human neuromodulation with transcranial focused ultrasound. J Neural Eng 14 : 066012, 2017

25. Na YC, Chang WS, Jung HH, Kweon EJ, Chang JW : Unilateral magnetic resonance-guided focused ultrasound pallidotomy for parkinson disease. Neurology 85 : 549-551, 2015
26. Sun J, Hynynen $\mathrm{K}$ : The potential of transskull ultrasound therapy and surgery using the maximum available skull surface area. J Acoust Soc Am 105 : 2519-2527, 1999

27. Tsai SJ : Transcranial focused ultrasound as a possible treatment for major depression. Med Hypotheses 84 : 381-383, 2015

28. Tung YS, Marquet F, Teichert T, Ferrera V, Konofagou EE : Feasibility of noninvasive cavitation-guided blood-brain barrier opening using focused ultrasound and microbubbles in nonhuman primates. Appl Phys Lett 98 : 163704, 2011

29. Wegener N, Kaegi G, Bauer R, Werner B, Martin E, Schreglmann SR, et al. : MR-guided high intensity focused ultrasound in Parkinson's disease: a series of 5 cases. Mov Disord 31 : S2, 2016

30. Wei KC, Chu PC, Wang HY, Huang CY, Chen PY, Tsai HC, et al. : Focused ultrasound-induced blood-brain barrier opening to enhance temozolomide delivery for glioblastoma treatment: a preclinical study. PLoS One 8 : e58995, 2013

31. Weintraub D, Elias WJ : The emerging role of transcranial magnetic resonance imaging-guided focused ultrasound in functional neurosurgery. Mov Disord 32 : 20-27, 2017

32. Wu SK, Chu PC, Chai WY, Kang ST, Tsai CH, Fan CH, et al. : Characterization of different microbubbles in assisting focused ultrasound-induced blood-brain barrier opening. Sci Rep 7 : 46689, 2017

33. Zaaroor M, Sinai A, Goldsher D, Eran A, Nassar M, Schlesinger I : Magnetic resonance-guided focused ultrasound thalamotomy for tremor: a report of 30 parkinson's disease and essential tremor cases. J Neurosurg $128: 202-210,2018$ 